\title{
Warped diffusive radio halo around the quiescent spiral edge-on galaxy NGC 4565
}

\author{
V. Heesen ${ }^{1}$, L. Whitler ${ }^{2}$, P. Schmidt ${ }^{3}$, A. Miskolczi ${ }^{4}$, S. S. Sridhar ${ }^{5}$, C. Horellou ${ }^{6}$, R. Beck ${ }^{3}$, G. Gürkan ${ }^{7}$, \\ E. Scannapieco ${ }^{2}$, M. Brüggen ${ }^{1}$, G. H. Heald ${ }^{7}$, M. Krause ${ }^{3}$, R. Paladino ${ }^{8}$, B. Nikiel-Wroczyński ${ }^{9}, 10$, \\ A. Wilber ${ }^{1}$, and R.-J. Dettmar ${ }^{4}$ \\ 1 University of Hamburg, Hamburger Sternwarte, Gojenbergsweg 112, 21029 Hamburg, Germany \\ e-mail: volker. heesen@hs. uni-hamburg. de \\ 2 School of Earth and Space Exploration, Arizona State University, PO Box 871404, Tempe, AZ 85287-1404, USA \\ 3 Max-Planck-Institute für Radioastronomie, Auf dem Hügel 69, 53121 Bonn, Germany \\ Astronomisches Institut der Ruhr-Universität Bochum, Universitätsstr. 150, 44780 Bochum, Germany \\ 5 ASTRON, the Netherlands Institute for Radio Astronomy, Postbus 2, 7990 AA Dwingeloo, The Netherlands \\ Chalmers University of Technology, Dept of Space, Earth and Environment, Onsala Space Observatory, 43992 Onsala, Sweden \\ CSIRO Astronomy and Space Science, PO Box 1130, Bentley, WA 6102, Australia \\ INAF/Istituto di Radioastronomia, Via Gobetti 101, 40129 Bologna, Italy \\ 9 Astronomical Observatory, Jagiellonian University, ul. Orla 171, 30-244 Kraków, Poland \\ ${ }^{10}$ Leiden Observatory, Leiden University, Oort Gebouw, PO Box 9513, 2300 RA Leiden, The Netherlands
}

Received 7 June 2019 / Accepted 12 July 2019

\begin{abstract}
Context. Cosmic rays play a pivotal role in launching galactic winds, particularly in quiescently star-forming galaxies where the hot gas alone is not sufficient to drive a wind. Except for the Milky Way, not much is known about the transport of cosmic rays in galaxies. Aims. In this Letter, we present low-frequency observations of the nearby edge-on spiral galaxy NGC 4565 using the LOw-Frequency ARray (LOFAR). With our deep $144 \mathrm{MHz}$ observations, we obtain a clean estimate of the emission originating from old cosmic-ray electrons (CRe), which is almost free from contamination by thermal emission.

Methods. We measured vertical profiles of the non-thermal radio continuum emission that we fitted with Gaussian and exponential functions. The different profile shapes correspond to $1 \mathrm{D}$ cosmic-ray transport models of pure diffusion and advection, respectively. Results. We detect a warp in the radio continuum that is reminiscent of the previously known Hi warp. Because the warp is not seen at GHz-frequencies in the radio continuum, its minimum age must be about $100 \mathrm{Myr}$. The warp also explains the slight flaring of the thick radio disc that can otherwise be well described by a Gaussian profile with an FWHM of $65 \operatorname{arcsec}(3.7 \mathrm{kpc})$.

Conclusions. The diffusive radio halo together with the extra-planar X-ray emission may be remnants of enhanced star-forming activity in the past where the galaxy had a galactic wind, as GHz-observations indicate only a weak outflow in the last 40 Myr. NGC 4565 could be in transition from an outflow- to an inflow-dominated phase.
\end{abstract}

Key words. galaxies: halos - galaxies: individual: NGC 4565 - galaxies: magnetic fields - galaxies: spiral - cosmic rays radio continuum: galaxies

\section{Introduction}

Radio haloes are a common feature around late-type spiral galaxies that are seen in an edge-on orientation (e.g. Irwin et al. 1999; Wiegert et al. 2015; Heesen et al. 2018a). The emission in the halo is dominated by the non-thermal radio continuum (synchrotron) component, which indicates cosmic ray electrons $(\mathrm{CRe})$ and magnetic fields. This is corroborated by the detection of linearly polarised emission, which allows us to study the structure of magnetic fields in the halo that often display a characteristic X-shape in projection (e.g. Tüllmann et al. 2000; Krause 2009; Wiegert et al. 2015). The structure of the magnetic field is of importance

* The LOFAR map and the integrated flux density from the LOFAR map are only available at the CDS via anonymous ftp to cdsarc.u-strasbg.fr (130.79.128.5) or via http://cdsarc. u-strasbg.fr/viz-bin/qcat?J/A+A/628/L3 for the transport of cosmic rays (protons, electrons, and heavier nuclei) from the disc into the halo. This is the scenario if cosmic rays are accelerated by diffusive shock acceleration in supernova (SN) remnants in the thin, star-forming galactic mid-plane (Bell 1978). This connection is corroborated by the tight correlation between radio continuum luminosity and star formation rate (SFR) in galaxies (e.g. Tabatabaei et al. 2017).

Cosmic ray transport can be described by two idealised modes, advection and diffusion, and the superposition of both. This distinction is important because advection-dominated haloes are indicative of outflows and winds. Outflows are directly observed over a wide range of galaxy masses, but in a smaller range of SFR surface densities. Large outflows are ubiquitous in galaxies in which the SFR density per unit area exceeds $\Sigma_{\mathrm{SFR}} \approx 0.1 M_{\odot} \mathrm{yr}^{-1} \mathrm{kpc}^{-2}$ (Heckman 2003), and they are more difficult to detect for lower rates (Chen et al. 2010). Observations also show that discs with strong outflows are characterised by 
velocity dispersions of $\sigma_{v}^{1 \mathrm{D}} \approx 50-100 \mathrm{~km} \mathrm{~s}^{-1}$ (e.g. Genzel et al. 2011; Swinbank et al. 2011), which occur at high surface densities. At these velocity dispersions, a thermal runaway occurs that facilitates the escape of supernova material from the galaxy (Scannapieco et al. 2012; Scannapieco 2013; Sur et al. 2016).

Advective haloes provide an avenue for detecting weaker outflows in environments with low SFR surface densities. This interpretation is suggested by the correlation between advection and rotational velocity of a galaxy, where the latter is a proxy for the escape velocity (Heesen et al. 2018a; Miskolczi et al. 2019; Schmidt et al. 2019). On the other hand, diffusive haloes allow us to measure diffusion coefficients and their energy dependencies, giving us measurements in addition to those in the Milky Way (Strong et al. 2007). Thus far, only one such example has been found in NGC 7462 (Heesen et al. 2016). This galaxy has an SFR surface density of $\Sigma_{\mathrm{SFR}}=1.6 \times 10^{-3} M_{\odot} \mathrm{yr}^{-1} \mathrm{kpc}^{-2}$, which is close to the threshold reported by Rossa \& Dettmar (2003) for the detection of extra-planar ionised gas (eDIG) in galaxies.

A threshold $\Sigma_{\text {SFR }}$ for advective haloes (and thus, galactic winds) is corroborated by a possible threshold for extra-planar $\mathrm{X}$-ray emission, which is a direct tracer of the hot SNe-heated gas. Tüllmann et al. (2006) found extra-planar X-ray emission only in galaxies with $\Sigma_{\mathrm{SFR}}>3 \times 10^{-3} M_{\odot} \mathrm{yr}^{-1} \mathrm{kpc}^{-2}$. If such a simple threshold exists, it will also depend on the distribution of star formation in the disc; galaxies in which star formation is more concentrated, for instance, nuclear starbursts, are more likely to have winds. Hydrodynamical simulations reported by Vasiliev et al. (2019) suggested a slightly higher SFR surface density for galaxy-wide outflows of $\Sigma_{\mathrm{SFR}} \approx 6 \times$ $10^{-3} M_{\odot} \mathrm{yr}^{-1} \mathrm{kpc}^{-2}$, but the authors did not take the dynamical importance of cosmic rays into account. Another key parameter that will influence any outflow or wind is its mass surface density (MSD; Krause et al. 2018), where high MSDs are expected to suppress outflows.

In this Letter, we present first results from our survey of nearby galaxies with the International LOw-Frequency ARray (LOFAR; van Haarlem et al. 2013), where we observed with the same setup as the LOFAR Two-metre Sky Survey (LoTSS; Shimwell et al. 2017). Low-frequency radio continuum observations have the benefit that we see the oldest CRe far away from star formation sites in the halo and that the contamination from thermal emission is minimal because the non-thermal spectrum rises steeply at low frequencies, whereas the thermal spectrum is essentially flat.

NGC 4565 is an Sbc late-type spiral galaxy at an assumed distance of $11.9 \mathrm{Mpc}$ (Tully \& Fisher 1988; Radburn-Smith et al. 2011), at an almost edge-on orientation with an inclination angle of 87.5 (Zschaechner et al. 2012), with a low $\Sigma_{\mathrm{SFR}}$ of $0.74 \times$ $10^{-3} M_{\odot} \mathrm{yr}^{-1} \mathrm{kpc}^{-2}$ (Wiegert et al. 2015). It has a total mass of $10^{11.4} M_{\odot}$, an MSD of $11.2 \times 10^{7} M_{\odot} \mathrm{kpc}^{-2}$, and an SFR of $0.73 M_{\odot} \mathrm{yr}^{-1}$ (Wiegert et al. 2015). This means that NGC 4565 is a quiescent galaxy, with both a low SFR and $\Sigma_{\mathrm{SFR}}$. It has an average MSD, in the middle of the range found in the sample of radio haloes studied by Krause et al. (2018), who covered a range between 4 and $16 \times 10^{8} M_{\odot} \mathrm{kpc}^{-2}$. This work is a low-frequency study of NGC 4565 complimentary to Schmidt et al. (2019), who presented the high-frequency $(1.5$ and $6 \mathrm{GHz})$ view. Radio spectral indices $\alpha$ are defined as $S_{v} \propto v^{\alpha}$, where $S_{v}$ is the flux density and $v$ is the observing frequency.

\section{Observations and data reduction}

Our High Band Antenna (HBA) LOFAR observations were taken on August 28 and October 12, 2017, as part of the LoTSS, where we observed the LoTSS pointing P194+27, which is 1.5 away from our target. We used the HBA-dual inner mode to conduct $8 \mathrm{~h}$ observations, with the $48 \mathrm{MHz}$ bandwidth (120-168 MHz), bookended by $10 \mathrm{~min}$ flux-calibrator scans (i.e. a total observing time of $8.3 \mathrm{~h})$. We stored the data at 16 channels per sub-band $(12.2 \mathrm{kHz}$ frequency resolution) and at $1 \mathrm{~s}$ time resolution. The data were reduced with the facet calibration technique, which mitigates the direction-dependent effects of the ionosphere and beam response that affect low-frequency radio continuum observations with aperture arrays, so that images close to the thermal noise level can be obtained (van Weeren et al. 2016; Williams et al. 2016). Below we describe the data reduction in some more detail.

First, the $(u, v)$ data were calibrated with direction-independent methods using the PREFACTOR pipeline (de Gasperin et al. 2019). Following the amplitude calibration using the flux densities of Scaife \& Heald (2012) for our primary calibrator, 3C 295, the station gain amplitudes and the phase variations due to the drift of the clocks and the Earth's ionosphere were determined. When they were determined, the instrumental calibration solutions were applied to the target data, which were then averaged to $10 \mathrm{~s}$ time resolution and a frequency resolution of twochannels per sub-band (channel width of $97.656 \mathrm{kHz}$ ). Then the data were calibrated in phase only using the global sky model (GSM; Scheers 2011). With the direction-independent calibration applied, the $(u, v)$ data were inverted and deconvolved with a wide-field CLEAN algorithm. As the final step of PREFACTOR, the CLEAN components of all the sources within the $8^{\circ}$ field of view (FoV) were subtracted from the $(u, v)$ data $^{1}$.

For the direction-dependent calibration we used the FACTOR pipeline $^{2}$. The FoV was divided into approximately 20 facets around calibrator regions with integrated $167 \mathrm{MHz}$ flux densities (of the full facet) in excess of $0.3 \mathrm{Jy}$. Of these, facets in excess of $0.8 \mathrm{Jy}$ were processed one at a time, beginning with the brightest facet. In the first step of the calibration, fast $10 \mathrm{~s}$ phase solutions were determined in small chunks of $\approx 2 \mathrm{MHz}$ bandwidth to correct for the positional change and distortion of sources. In the second step, slow long-amplitude (tens of minutes) solutions were used to track the variation in the apparent flux density of a source. The target facets were corrected using the solution of a nearby facet.

The direction-dependent calibrated $(u, v)$ data were imported into the Common Astronomy Software Applications (CASA; McMullin et al. 2007) and inverted and deconvolved with the MS-MFS CLEAN algorithm (Rau \& Cornwell 2011). We fitted for the frequency dependence of the skymodel $($ nterms $=2$ ) and used angular scales of up to 88 arcsec for the deconvolution. This is significantly smaller than the size of the galaxy ( 16 arcmin), but we found that increasing the largest angular scale does result in large residual areas of negative flux densities above and below the galaxy. We verified that in spite of the small angular scale no significant flux was left in the residual map. We used Briggs weighting, setting the robust parameter to 0.2 , which resulted in a map with an effective central frequency of $144 \mathrm{MHz}$ with angular resolutions of 20 arcsec FWHM with a map noise of $\sigma=130 \mu \mathrm{Jy} \mathrm{beam}^{-1}$. The radio continuum spectrum of this galaxy is discussed in Appendix A.

\section{Results and discussion}

Figure 1 shows the radio continuum emission at $144 \mathrm{MHz}$ displayed as contours on an optical image from the Sloan Digital

\footnotetext{
https://github.com/lofar-astron/prefactor https://github.com/lofar-astron/factor
} 


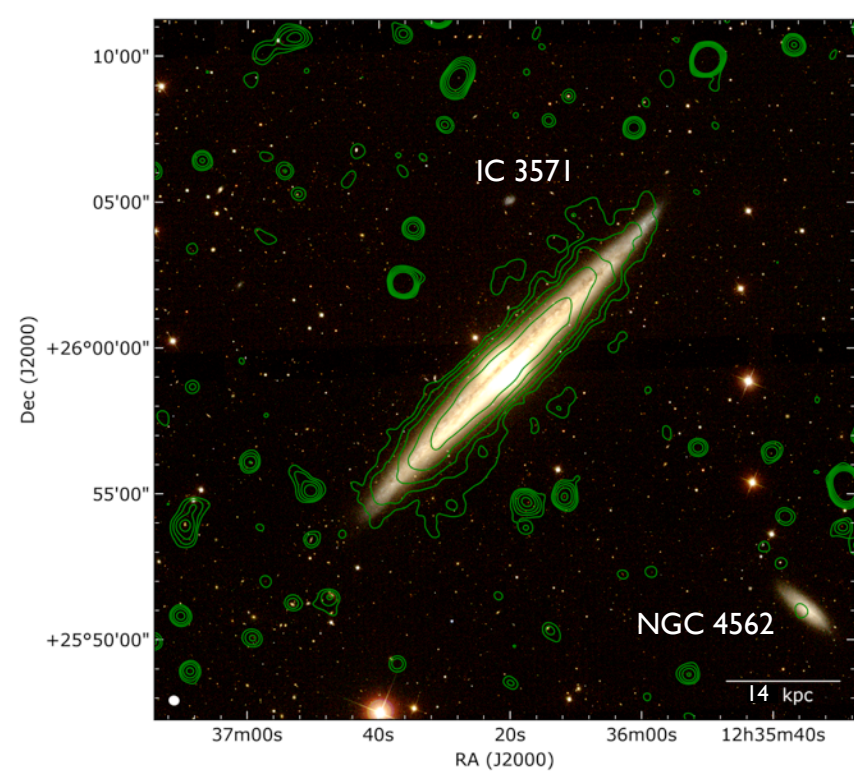

Fig. 1. Radio continuum emission at $144 \mathrm{MHz}$, presented as contours on an SDSS image, at $19.6 \times 17.5 \operatorname{arcsec}^{2}$ FWHM resolution. Contours start at a level of $3 \sigma\left(390 \mu \mathrm{Jy}_{\text {beam }}^{-1}\right)$, increasing by a factor of 2 . The size of the synthesised beam is shown in the bottom left corner in white.

Sky Survey (SDSS; York et al. 2000). It is striking that the emission is almost purely confined to the optical disc with very little extra-planar emission. The extent of the first contour along the major axis is 14 arcmin, but the extent along the minor axis is only 2.2 arcmin in the centre of the galaxy, increasing to 2.9 arcmin south-east of the centre. This galaxy therefore has a major-to-minor-axis ratio of around 5. This value can be compared with the value reported by Singal et al. (2015), who found in their sample of ten spiral edge-on galaxies a ratio of $1.4 \pm 0.5$, similarly to Wiegert et al. (2015), who stacked 30 of $1.5 \mathrm{GHz}$ images. In dwarf irregular galaxies, the ratio can even be lower than unity (Heesen et al. 2018b). Clearly, the axis ratio of the contours depends on frequency, angular resolution, and sensitivity, but the stark contrast already shows that NGC 4565 is quite different from the galaxies that were studied so far.

A more quantitative way to measure radio halo properties is to use vertical profiles of the non-thermal intensities, for which we have subtracted the thermal emission from a combination of $\mathrm{H} \alpha$ and Spitzer $24 \mu \mathrm{m}$ emission (adopted from Schmidt et al. 2019). This correction is small: the thermal fraction is only $2 \%$ at $144 \mathrm{MHz}$, with most of the emission concentrated in the thin star-forming disc, so that the thermal fraction in the halo is entirely negligible. In Fig. 2 we present these profiles, which we measured with BoxModels (Müller et al. 2017), and fitted them with one-component Gaussian and exponential fits. The results are presented in Table 1 . It is immediately clear that they cannot be fitted with one-component exponential profiles, except possibly in the outer two profiles (strips a and e, because of their large error bars), where we find $1.1<\chi_{\text {red }}^{2}<21.5$. In contrast, the profiles can be very well fitted by single-component Gaussian functions $\left(0.34<\chi_{\text {red }}^{2}<0.43\right)$, with an FWHM that increases from 61.4 arcsec in the central stripe to 71.9 arcsec in the north-western, outer stripe (strip e) and similarly in the south-eastern, outer stripe (strip a). These measured extents are well resolved (we recall the angular resolution of 20 arcsec). We therefore detect a slight flaring of the radio halo, as suggested by the shape of the radio emission contours. This is also seen at higher frequencies (Schmidt et al. 2019).
For the first time, we can report a warp in the radio continuum (compare with Fig. 1) that is reminiscent of the H I warp in the outer parts of the galaxy. The H I warp can be seen in projection against the inner disc (Zschaechner et al. 2012). The flaring of the radio halo seems to be caused by the warp because the vertical intensity profiles (with the exception of the central stripe) are asymmetric, in agreement with the warp. We can therefore now give a minimum age for the warp of approximately $130 \mathrm{Myr}$, which is the spectral age of the CRe during which they are transported into the warp. The warp is not visible at $\mathrm{GHz}$ frequencies (Heesen et al. 2018a; Schmidt et al. 2019), which means that we can rule out a younger age of $40 \mathrm{Myr}$ or less. Radburn-Smith et al. (2014) found young ( $<600 \mathrm{Myr})$ stars in the warp with a lack of older $(>1 \mathrm{Gyr})$ stars, which places an upper limit on the age of the structure.

We fitted the intensity profiles with $1 \mathrm{D}$ cosmic ray transport models for advection and diffusion using SPINNAKER (Heesen et al. 2016). In order to calculate the radio spectral indices that are required as input, we used a $1570 \mathrm{MHz}$ map from $L$ band observations with the Karl G. Jansky Very Large Array (JVLA), combined from the B, C, and D array (Schmidt et al. 2019). These data were taken as part of the "Continuum Halos in Nearby Galaxies, an EVLA Survey" (CHANG-ES; Irwin et al. 2012). The resulting spectral index map is shown in Fig. 3. In the galactic mid-plane, relatively flat spectral indices $\alpha \approx-0.7$ are found, which are indicative of young CRe. In contrast, the steep spectral indices of $\alpha \approx-0.9$ in the halo are indicative of old CRe. This is the expected behaviour if young CRe are injected at star formation sites in the mid-plane, from where they are transported into the halo. We therefore fitted the profiles except in the central stripe c, which might be influenced by an outflow from the Seyfert nucleus (Ho et al. 1997).

Our model assumes that the CRe are injected with a power law in the galactic mid-plane and lose their energy through synchrotron and inverse Compton losses. A steady state between injection and losses is then reached. We present the best-fitting parameters for these models in Table 2 and the corresponding profiles in Appendix B. We found that pure CRe diffusion can describe our $144 \mathrm{MHz}$ data better than advection, where the reduced $\chi^{2}$ is on average a factor of two smaller for diffusion. On the other hand, advection can describe the $1570 \mathrm{MHz}$ profiles better than diffusion; the improvement is even more pronounced. This outcome can be explained if the galaxy is in the aftermath of a period with more intense star formation, as has been proposed by Schmidt et al. (2019). The LOFAR map is then dominated by the CRe that were injected during a past starburst and had time to slowly diffuse away from star formation sites in the galactic mid-plane. In contrast, the emitting $\mathrm{CRe}$ at $\mathrm{GHz}$ frequencies are dominated by those that are advected in the current weak outflow.

This scenario is corroborated by several observations. The mid- and far-infrared emission from dust together with radiative transfer modelling suggest that the SFR has indeed declined in the past $100 \mathrm{Myr}$ (De Looze et al. 2012), which is in good agreement with the CRe lifetime at the LOFAR frequency. Furthermore, there is a reservoir of extra-planar X-ray emission in the halo that extends up to $10 \mathrm{kpc}$ away from the galactic mid-plane (see Fig. 4). This emission is usually thought to be a remnant of a past starburst. At $1570 \mathrm{MHz}$, the vertical profiles of the nonthermal radio continuum can be described by two-component

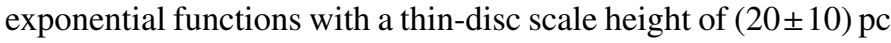
(Schmidt et al. 2019); this means that the thin disc is much less extended than in other galaxies, where it has scale height of around $400 \mathrm{pc}$ (Heesen et al. 2018a). The data are consistent with either diffusion or advection in the halo (height $>1 \mathrm{kpc}$ ), but 

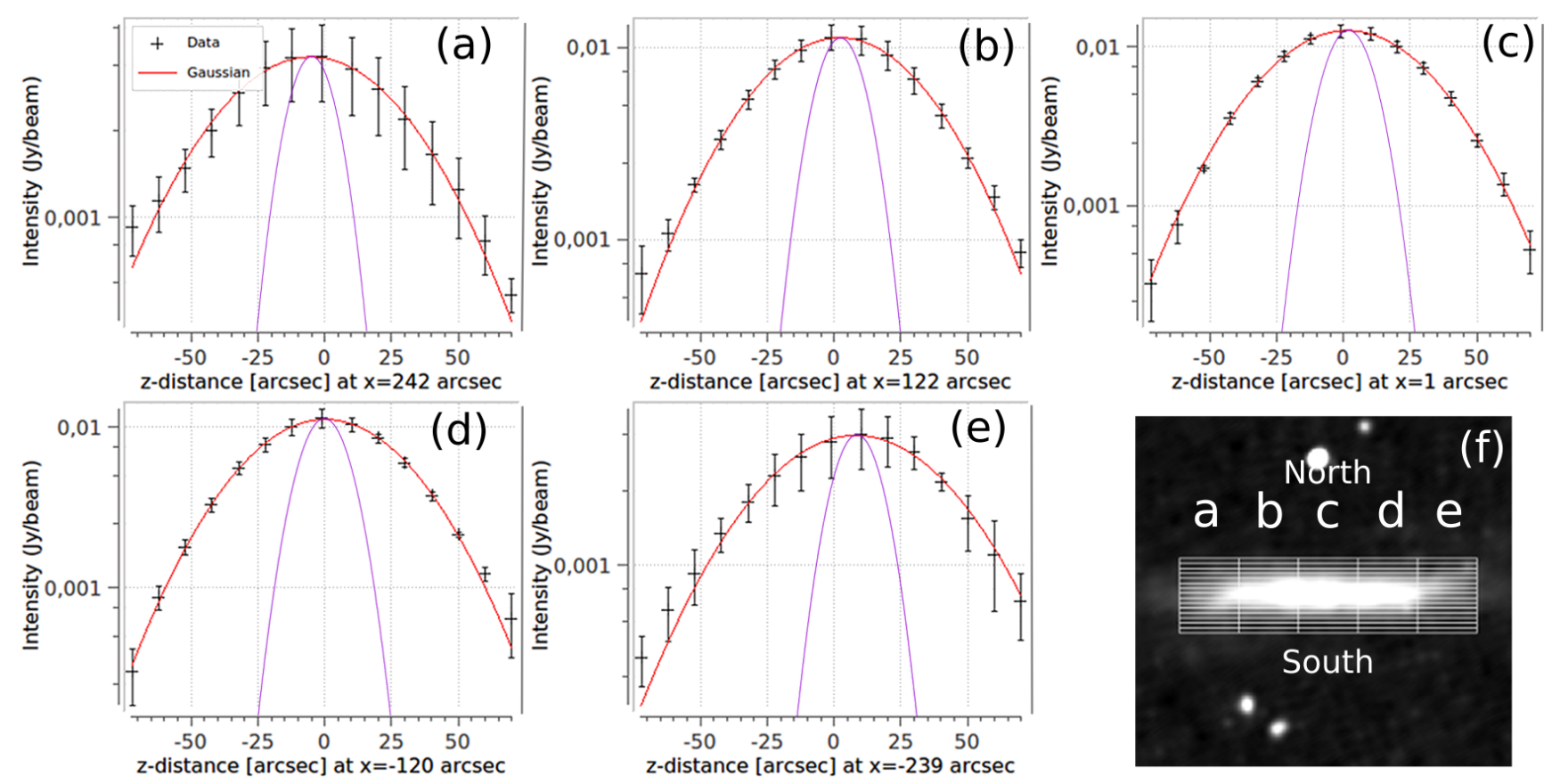

Fig. 2. Vertical profiles of the non-thermal radio continuum emission at $144 \mathrm{MHz}$ in strips at various offsets along the major axis (panels a-e) and the position of the strips (panel f). Red solid lines show Gaussian model fits to the data, and purple solid lines show the Gaussian synthesised beam $(F W H M=20$ arcsec $)$. Vertical tick marks are at $2.5 \times, 5 \times$, and $7.5 \times$ the next labelled tick value below. In the profiles, south is to the left and north is to the right; 1 arcsec corresponds to $57 \mathrm{pc}$ at the distance of NGC 4565.

Table 1. Best-fitting Gaussian and exponential one-component fits.

\begin{tabular}{ccccc}
\hline \hline Strip & $\begin{array}{c}F W H M \\
(\operatorname{arcsec})\end{array}$ & $\begin{array}{c}\text { Gauss } \\
\chi_{\text {red }}^{2}\end{array}$ & $\begin{array}{c}h \\
(\operatorname{arcsec})\end{array}$ & $\begin{array}{c}\text { Exp. } \\
\chi_{\text {red }}^{2}\end{array}$ \\
\hline (a) & $71.87 \pm 1.42$ & 0.37 & $33.45 \pm 1.96$ & 1.93 \\
(b) & $64.04 \pm 0.75$ & 0.48 & $28.58 \pm 1.78$ & 4.59 \\
(c) & $61.41 \pm 0.27$ & 0.34 & $26.99 \pm 2.17$ & 21.53 \\
(d) & $60.94 \pm 0.55$ & 0.43 & $26.71 \pm 1.79$ & 11.09 \\
(e) & $70.42 \pm 1.73$ & 0.34 & $32.74 \pm 2.33$ & 1.06 \\
\hline
\end{tabular}

with an outflow speed lower than the escape velocity. If an outflow currently exists, it is probably a fountain flow rather than a wind.

We found mostly good fits to the data with the exception of strip d north, where neither diffusion nor advection leads to good fits. This strip is in the region of the Hi bridge that connects NGC 4565 with its satellite galaxy IC 3571 (RadburnSmith et al. 2014, see also Fig. 1). It is therefore possible that tidal interaction governs the gas dynamics in this area and the transport of CRe with it, such that our idealised cosmic ray transport models no longer fit. We find diffusion coefficients that are in good agreement with Schmidt et al. (2019) and the canonical Milky Way value of $3 \times 10^{28} \mathrm{~cm}^{2} \mathrm{~s}^{-1}$ (Strong et al. 2007). We mostly failed to find any energy dependence, just as Schmidt et al. (2019) found at higher frequencies. While they were unable to formally distinguish between diffusion and advection, however, our new LOFAR data require diffusion to be the dominant transport process.

\section{Conclusions}

We have observed the nearby edge-on spiral galaxy NGC 4565 with LOFAR at $144 \mathrm{MHz}$ in the radio continuum to measure the

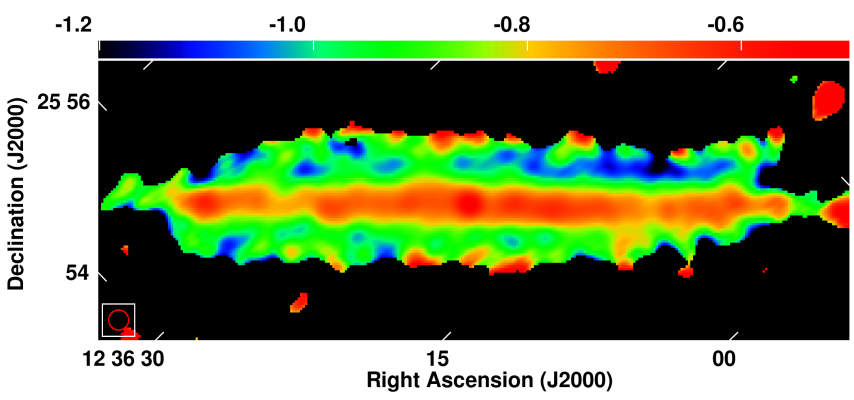

Fig. 3. Non-thermal radio spectral index between 144 (LOFAR) and $1570 \mathrm{MHz}$ (JVLA) at 20 arcsec FWHM resolution. The major axis has been rotated so that it is horizontal. The size of the synthesised beam is shown in the bottom left corner.

distribution of $\mathrm{CRe}$ and magnetic fields. Our results are listed below.

(i) The radio continuum emission is constrained to a very elongated thin distribution with a major-to-minor-axis ratio of around 5.

(ii) The vertical intensity profiles can be well fitted by a onecomponent Gaussian function, which is the first case of such at low frequencies. Our explanation is that NGC 4565 has an old $(\approx 100 \mathrm{Myr}$ ) population of $\mathrm{CRe}$ that has slowly diffused away from the star-forming disc.

(iii) There are indications that NGC 4565 is in the aftermath of a period with more intense star formation. The weak outflow that may be seen at higher frequencies was therefore probably stronger in the past, which explains the diffusive low-frequency radio halo.

Outflows and inflows in galaxies are thought to occur in quasi-periodic fashion. Bursts of star formation lead to outflows that remove gas from the disc into the halo. This gas, in particular the warm component, will then fall back onto the disc as part of a so-called fountain flow during periods of less intense star 
Table 2. Best-fitting diffusion and advection models.

\begin{tabular}{lcccc}
\hline \hline Strip & $\begin{array}{c}\text { LOFAR } \\
\chi_{\text {red }}^{2} \\
\text { (diff/adv) }\end{array}$ & $\begin{array}{c}\text { JVLA } \\
\chi_{\text {red }}^{2} \\
\text { (diff/adv) }\end{array}$ & $\begin{array}{c}D_{0} \\
\left(10^{28} \mathrm{~cm}^{2} \mathrm{~s}^{-1}\right)\end{array}$ & $\begin{array}{c}V \\
\left(\mathrm{~km} \mathrm{~s}^{-1}\right)\end{array}$ \\
\hline (a) $\mathrm{N}$ & $0.05 / 0.07$ & $0.38 / 0.09$ & $1.5_{-0.8}^{+8.5}(\mu=0.4)$ & $66_{-30}^{+180}$ \\
(a) $\mathrm{S}$ & $0.15 / 0.24$ & $0.21 / 0.08$ & $2.3_{-1.0}^{+1.9}(\mu=0.0)$ & $56_{-20}^{+40}$ \\
(b) $\mathrm{N}$ & $0.74 / 1.16$ & $1.50 / 0.38$ & $3.1_{-0.9}^{+1.7}(\mu=0.0)$ & $82_{-15}^{+30}$ \\
(b) $\mathrm{S}$ & $0.40 / 0.92$ & $1.84 / 0.54$ & $2.1_{-0.6}^{+1.1}(\mu=0.0)$ & $67_{-10}^{+20}$ \\
(d) $\mathrm{N}$ & $1.55 / 5.16$ & $6.96 / 2.26$ & $1.5_{-0.5}^{+0.9}(\mu=0.0)$ & $54_{-15}^{+30}$ \\
(d) $\mathrm{S}$ & $0.76 / 3.31$ & $0.69 / 0.13$ & $0.7_{-0.1}^{+0.2}(\mu=0.5)$ & $81_{-20}^{+60}$ \\
(e) $\mathrm{N}$ & $0.64 / 0.70$ & $0.63 / 0.17$ & $3.3_{-1.6}^{9.0}(\mu=0.0)$ & $66_{-25}^{+90}$ \\
(e) $\mathrm{S}$ & $0.04 / 0.07$ & $0.15 / 0.05$ & $1.6_{-0.8}^{+2.5}(\mu=0.0)$ & $46_{-25}^{+50}$ \\
\hline
\end{tabular}

Notes. Reduced $\chi^{2}$, diffusion coefficients $\left(D=D_{0}(E / \mathrm{GeV})^{\mu}\right)$, and advection speeds $(V)$ in the northern $(\mathrm{N})$ and southern $(\mathrm{S})$ haloes.

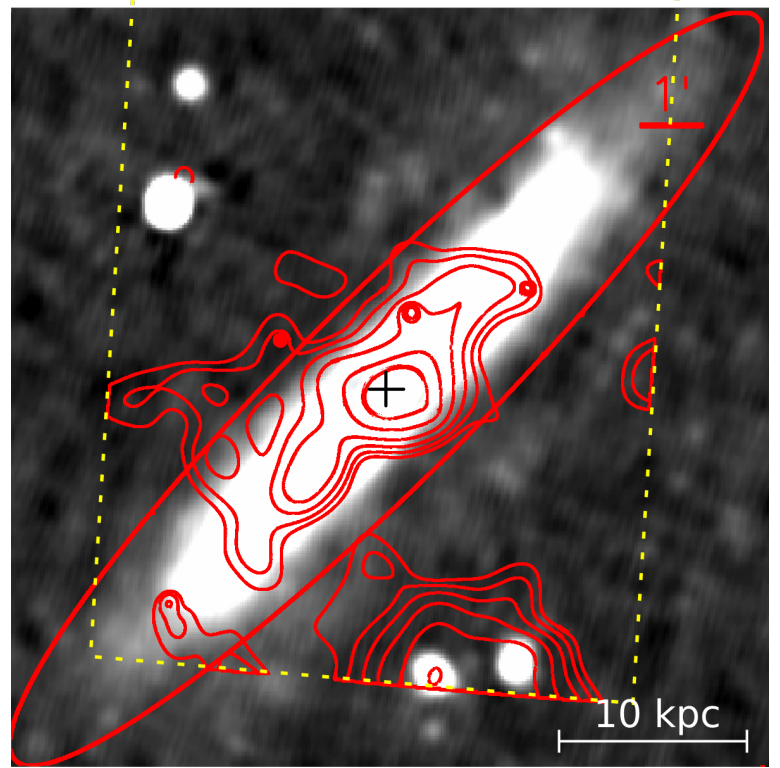

Fig. 4. Soft X-ray emission (0.5-1.5 keV) as contours (Li \& Wang 2013, see their Fig. 4 for details), overlaid on $144 \mathrm{MHz}$ radio continuum emission as grey scale $\left(-0.6\right.$ to $\left.3 \mathrm{mJy} \mathrm{beam}^{-1}\right)$ at 20 arcsec FWHM resolution. The ellipse shows the $D_{25}$ optical diameter ( $\left.16.7 \mathrm{arcmin}\right)$, the position of the nucleus is marked by a cross, and the yellow dashed line shows the Chandra FoV.

formation (Kim \& Ostriker 2018). NGC 4565 may be an example of a galaxy that we observe in a transition state from an outflow to an inflow dominated period. This would explain why it behaves differently to NGC 7462, the only other galaxy in which we have discovered a diffusive radio halo so far. There, the GHzprofiles can be fitted by a one-component Gaussian fit, such that the galaxy would already be in the inflow-dominated phase and would entirely lack an outflow.

When we visualize galactic winds in our minds, the local starburst galaxy M 82 is arguably the most commonly used picture (e.g. Leroy et al. 2015). However, the evidence is now mounting that outflows and winds play a crucial role even in the evolution of fairly quiescent galaxies; for instance, the stellar population synthesis analysis of NGC 628 required the efficient removal of mass as a result of stellar feedback (Zaragoza-Cardiel et al. 2019). Radio continuum observations with LOFAR open up a new window on the population of cosmic rays in these sys- tems, allowing us to study their significance for galaxy evolution in much more detail.

Acknowledgements. We would like to thank the anonymous referee for an insightful report. We also thank Hans-Rainer Klöckner for carefully reading the manuscript. LOFAR, the Low Frequency Array designed and constructed by ASTRON, has facilities in several countries that are owned by various parties (each with their own funding sources) and that are collectively operated by the International LOFAR Telescope (ILT) foundation under a joint scientific policy. Funding for the Sloan Digital Sky Survey IV has been provided by the Alfred P. Sloan Foundation, the U.S. Department of Energy Office of Science, and the Participating Institutions. LW and ES were supported by the NSF under grant OSIE-1458445.

\section{References}

Bell, A. R. 1978, MNRAS, 182, 147

Chen, H.-W., Helsby, J. E., Gauthier, J.-R., et al. 2010, ApJ, 714, 1521 de Gasperin, F., Dijkema, T. J., Drabent, A., et al. 2019, A\&A, 622, A5 De Looze, I., Baes, M., Bendo, G. J., et al. 2012, MNRAS, 427, 2797 Genzel, R., Newman, S., Jones, T., et al. 2011, ApJ, 733, 101

Heckman, T. M. 2003, in Rev. Mex. Astron. Astrof. Conf. Ser., eds. V. AvilaReese, C. Firmani, C. S. Frenk, \& C. Allen, 17, 47

Heeschen, D. S., \& Wade, C. M. 1964, AJ, 69, 277

Heesen, V., Dettmar, R.-J., Krause, M., Beck, R., \& Stein, Y. 2016, MNRAS, 458,332

Heesen, V., Krause, M., Beck, R., et al. 2018a, MNRAS, 476, 158

Heesen, V., Rafferty, D. A., Horneffer, A., et al. 2018b, MNRAS, 476, 1756

Ho, L. C., Filippenko, A. V., \& Sargent, W. L. W. 1997, ApJS, 112, 315

Irwin, J. A., English, J., \& Sorathia, B. 1999, AJ, 117, 2102

Irwin, J., Beck, R., Benjamin, R. A., et al. 2012, AJ, 144, 43

Kim, C.-G., \& Ostriker, E. C. 2018, ApJ, 853, 173

Krause, M. 2009, Rev. Mex. Astron. Astrofis., 36, 25

Krause, M., Irwin, J., Wiegert, T., et al. 2018, A\&A, 611, A72

Leroy, A. K., Walter, F., Martini, P., et al. 2015, ApJ, 814, 83

Li, J.-T., \& Wang, Q. D. 2013, MNRAS, 428, 2085

McMullin, J. P., Waters, B., Schiebel, D., Young, W., \& Golap, K. 2007, in Astronomical Data Analysis Software and Systems XVI, eds. R. A. Shaw, F. Hill, \& D. J. Bell (San Francisco: Astron. Soc. Pac.), ASP Conf. Ser., 376, 127

Miskolczi, A., Heesen, V., Horellou, C., et al. 2019, A\&A, 622, A9

Müller, P., Krause, M., Beck, R., \& Schmidt, P. 2017, A\&A, 606, A41

Niklas, S., Klein, U., Braine, J., \& Wielebinski, R. 1995, A\&AS, 114, 21

Radburn-Smith, D. J., de Jong, R. S., Seth, A. C., et al. 2011, ApJS, 195, 18

Radburn-Smith, D. J., de Jong, R. S., Streich, D., et al. 2014, ApJ, 780, 105

Rau, U., \& Cornwell, T. J. 2011, A\&A, 532, A71

Rossa, J., \& Dettmar, R.-J. 2003, A\&A, 406, 493

Scaife, A. M. M., \& Heald, G. H. 2012, MNRAS, 423, L30

Scannapieco, E. 2013, ApJ, 763, L31

Scannapieco, E., Gray, W. J., \& Pan, L. 2012, ApJ, 746, 57

Scheers, L. H. A. 2011, PhD Thesis, Univ. Amsterdam

Schmidt, P., Krause, M., Heesen, V., et al. 2019, A\&A, accepted https://doi.org/10.1051/0004-6361/201834995

Shimwell, T. W., Röttgering, H. J. A., Best, P. N., et al. 2017, A\&A, 598, A104

Singal, J., Kogut, A., Jones, E., \& Dunlap, H. 2015, ApJ, 799, L10

Strong, A. W., Moskalenko, I. V., \& Ptuskin, V. S. 2007, Annu. Rev. Nucl. Part. Sci., 57, 285

Sur, S., Scannapieco, E., \& Ostriker, E. C. 2016, ApJ, 818, 28

Swinbank, A. M., Papadopoulos, P. P., Cox, P., et al. 2011, ApJ, 742, 11

Tabatabaei, F. S., Schinnerer, E., Krause, M., et al. 2017, ApJ, 836, 185

Tüllmann, R., Dettmar, R.-J., Soida, M., Urbanik, M., \& Rossa, J. 2000, A\&A, 364, L36

Tüllmann, R., Pietsch, W., Rossa, J., Breitschwerdt, D., \& Dettmar, R.-J. 2006, A\&A, 448, 43

Tully, R. B., \& Fisher, J. R. 1988, Catalog of Nearby Galaxies (Cambridge: Cambridge University Press)

van Haarlem, M. P., Wise, M. W., Gunst, A. W., et al. 2013, A\&A, 556, A2 van Weeren, R. J., Williams, W. L., Hardcastle, M. J., et al. 2016, ApJS, 223, 2 Vasiliev, E. O., Shchekinov, Y. A., \& Nath, B. B. 2019, MNRAS, 486, 3685 Wiegert, T., Irwin, J., Miskolczi, A., et al. 2015, AJ, 150, 81

Williams, W. L., van Weeren, R. J., Röttgering, H. J. A., et al. 2016, MNRAS, 460, 2385

York, D. G., Adelman, J., Anderson, J. E., et al. 2000, AJ, 120, 1579

Zaragoza-Cardiel, J., Fritz, J., Aretxaga, I., et al. 2019, MNRAS, 487, L61

Zschaechner, L. K., Rand, R. J., Heald, G. H., Gentile, G., \& Józsa, G. 2012, ApJ, 760, 37 


\section{Appendix A: Radio continuum spectrum}

Table A.1. Integrated radio continuum flux densities of NGC 4565.

\begin{tabular}{lcc}
\hline \hline $\begin{array}{l}v \\
(\mathrm{GHz})\end{array}$ & $\begin{array}{c}S_{v} \\
(\mathrm{Jy})\end{array}$ & Reference \\
\hline 0.144 & $1.01 \pm 0.10$ & This work \\
0.750 & $0.30 \pm 0.03$ & Heeschen \& Wade (1964) \\
1.57 & $0.146 \pm 0.007$ & Schmidt et al. (2019) \\
6.0 & $0.0482 \pm 0.0039$ & Schmidt et al. (2019) \\
10.55 & $0.028 \pm 0.003$ & Niklas et al. (1995) \\
\hline
\end{tabular}

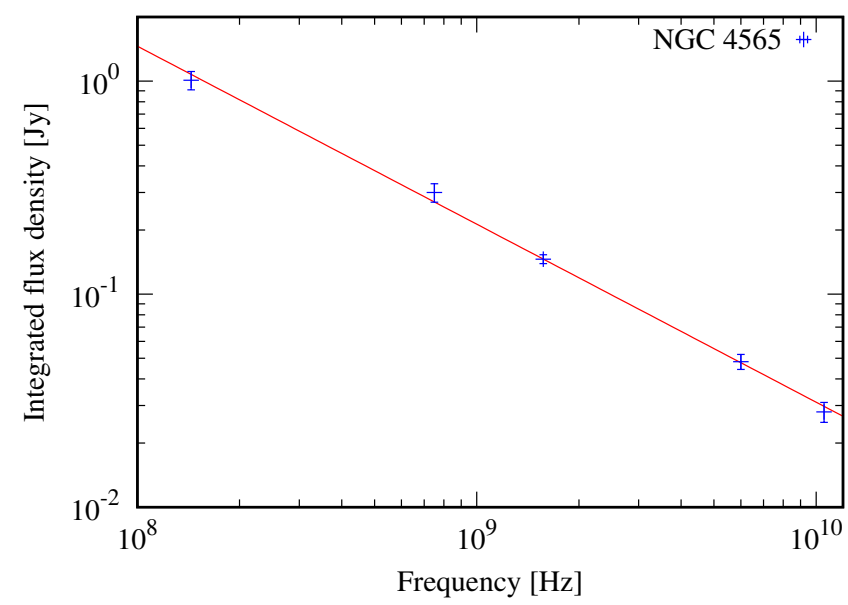

Fig. A.1. Radio continuum spectrum of NGC 4565. The integrated flux densities are shown as data points, and the solid line is the best-fitting power law.

In this appendix, we present the integrated radio continuum spectrum of NGC 4565 . We integrated the $144 \mathrm{MHz}$ flux density in an area approximately encompassing the $3 \sigma$ contour line, resulting in $1.01 \pm 0.10 \mathrm{Jy}$. The error of $10 \%$ was adopted from Shimwell et al. (2017). This error is larger than the primary calibrator model uncertainty of approximately $3 \%$ (Scaife \& Heald 2012) because the uncertainty in the beam model affects observations with aperture arrays of fixed dipoles. Literature flux densities are presented in Table A.1. The radio continuum spectrum of NGC 4565 is therefore a power-law between $144 \mathrm{MHz}$ and $10.55 \mathrm{GHz}$ with a spectral index of $-0.836 \pm 0.022\left(\chi_{\text {red }}^{2} \equiv\right.$ $\chi^{2} /$ d.o.f. $\left.=0.6\right)$. The resulting radio continuum spectrum together with the best-fitting power law is presented in Fig. A.1.

\section{Appendix B: Cosmic ray transport models}

In this appendix, we present the best-fitting advection and diffusion models for each strip. We assume that there is a steady state
Table B.1. Further parameters used during the modelling with SPINNAKER.

\begin{tabular}{lccc}
\hline \hline Strip & $B_{0}$ & $\begin{array}{c}h_{\mathrm{B}} \\
(\text { diff/adv) } \\
(\mathrm{kpc})\end{array}$ & $\begin{array}{c}\gamma \\
\text { (diff/adv) }\end{array}$ \\
\hline (a) $\mathrm{N}$ & 5.2 & $3.4_{-1.4}^{+2.2} / 3.4_{-1.4}^{+1.5}$ & $2.6 / 2.4$ \\
(a) $\mathrm{S}$ & 5.2 & $4.9_{-2.0}^{+3.0} / 4.5_{-1.7}^{+2.8}$ & $2.5 / 2.4$ \\
(b) $\mathrm{N}$ & 6.4 & $3.5_{-0.7}^{+0.7} / 3.4_{-0.5}^{+0.5}$ & $2.5 / 2.5$ \\
(b) $\mathrm{S}$ & 6.4 & $2.9_{-0.5}^{+0.5} / 2.7_{-0.3}^{+0.3}$ & $2.4 / 2.4$ \\
(d) $\mathrm{N}$ & 6.4 & $3.6_{-0.8}^{+1.0} / 3.1_{-0.5}^{+0.5}$ & $2.5 / 2.5$ \\
(d) $\mathrm{S}$ & 6.4 & $3.2_{-0.6}^{+0.5} / 2.5_{-0.3}^{+0.3}$ & $2.4 / 2.4$ \\
(e) $\mathrm{N}$ & 5.4 & $6.0_{-2.3}^{+6.3} / 6.1_{-3.0}^{+7.5}$ & $2.7 / 2.6$ \\
(e) $\mathrm{S}$ & 5.4 & $3.6_{-1.5}^{+2.0} / 3.2_{-1.2}^{+1.5}$ & $2.6 / 2.4$ \\
\hline
\end{tabular}

Notes. The magnetic field strength in the galactic mid-plane, $B_{0}$, is an input. The magnetic field scale height $h_{\mathrm{B}}$ is fitted during the modelling, and so is the CRe injection spectral index $\gamma$.

between CRe injection in the galactic mid-plane and CRe losses through synchrotron and inverse Compton radiation. As input parameters we used the equipartition magnetic field strength in the disc plane from Schmidt et al. (2019). We used $U_{\mathrm{IRF}} / U_{\mathrm{B}}=$ 0.35 as the ratio of the energy densities of the interstellar radiation field (IRF) to that of the magnetic field. This means that synchrotron losses of the CRe dominate inverse Compton losses. We used SPINNAKER (Heesen et al. 2016, 2018a) to calculate the 1D cosmic ray transport models for pure diffusion and advection with the fitting carried out in SPINTERACTIVE (A. Miskolczi 2018, priv. comm. ${ }^{3}$ ).

The CRe were injected in the galactic mid-plane with a power-law $N=N_{0} E^{-\gamma}$, where the CRe injection spectral index $\gamma$ is a free parameter. The magnetic field strength in the halo was parametrised by an exponential distribution in the halo, with $B(z)=B_{0} \exp \left(-z / h_{\mathrm{B}}\right)$, where $z$ is the distance to the disc and $h_{\mathrm{B}}$ is the magnetic field scale height. The magnetic field scale height was fitted with the resulting scale heights presented in Table B.1; values are between 2.5 and $6.1 \mathrm{kpc}$, where the best-fitting scale height is similar for diffusion and advection. We find that the scale heights in the different strips agree within the uncertainties. The maximum scale height, although still not significant when the errors are considered, is found in strip e (north), with the second highest scale height found in strip a (south). This is in agreement with the position of the warp that is visible in the radio continuum emission.

In Figs. B.1-B.3 we show the best-fitting models in the four strips in which we fitted the models (excluding the central strip). We treated the northern and southern haloes separately, therefore we fitted eight different profiles each with pure diffusion and advection, so that there are 16 different panels. 
Diffusion

(a) north

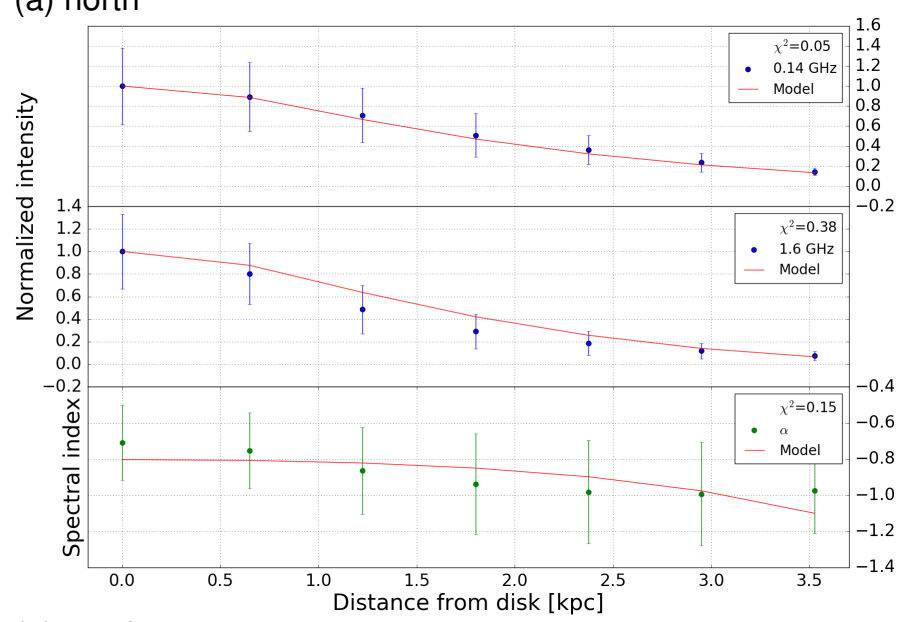

(a) south

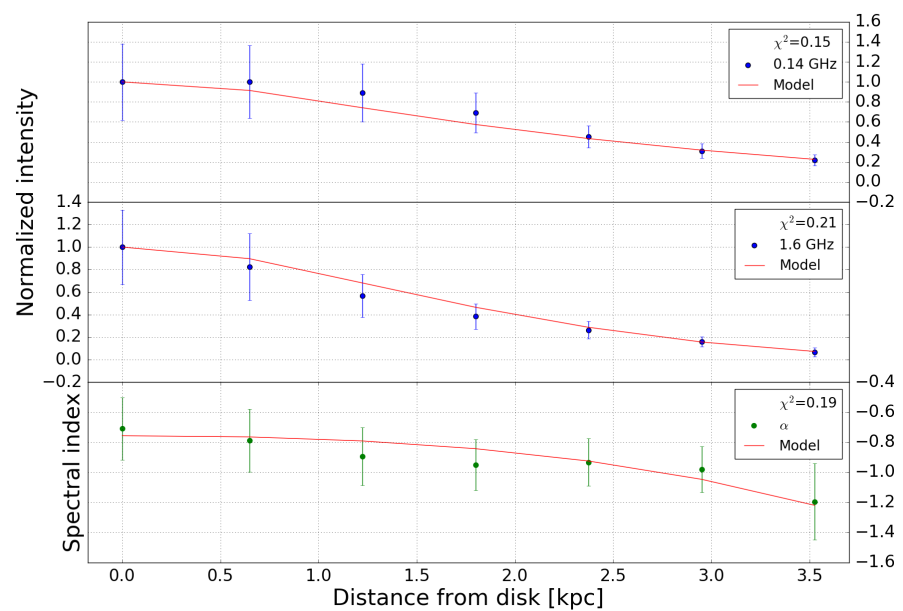

(b) north

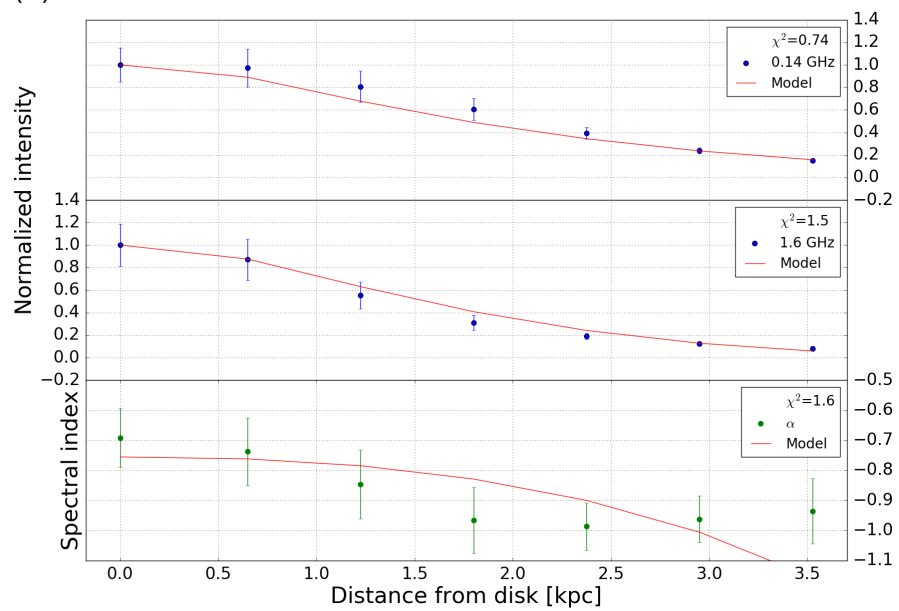

(a) north

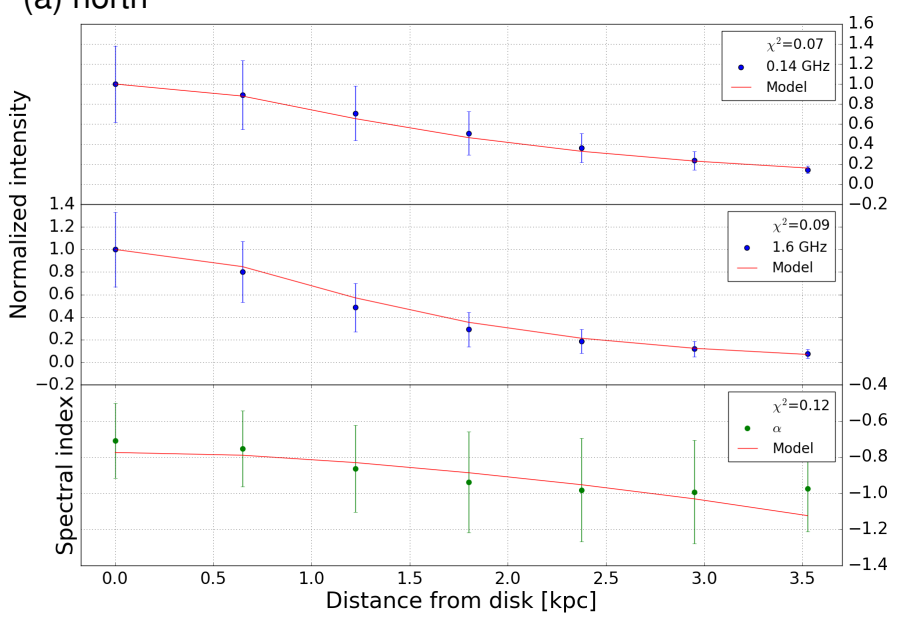

(a) south

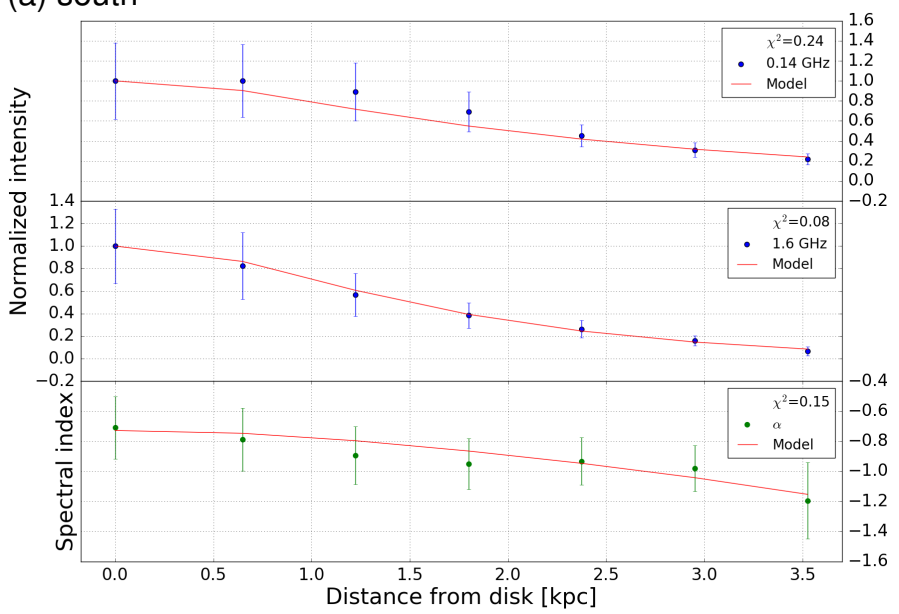

(b) north

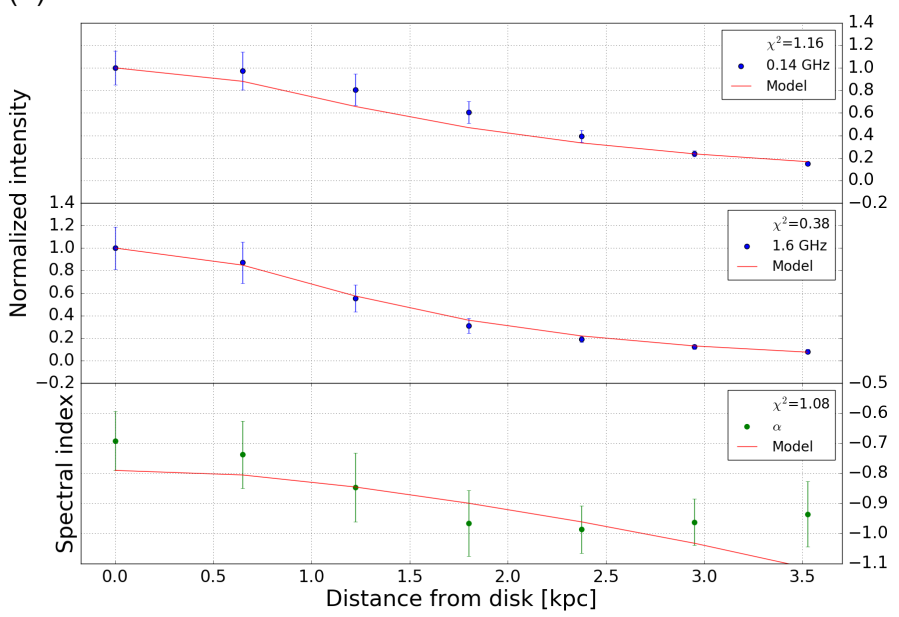

Fig. B.1. Best-fitting diffusion (left panels) and advection (right panels) models in strips a (north and south) and b (north). In each panel we show the vertical profiles. From top to bottom: $144 \mathrm{MHz}$ non-thermal intensities, the $1570 \mathrm{MHz}$ non-thermal intensities, and the non-thermal radio spectral index. Solid lines show the best-fitting models calculated with SPINNAKER. In each panel we also present the reduced $\chi_{\text {red }}^{2}$ for each observed profile and model profile. 
Diffusion

(b) south

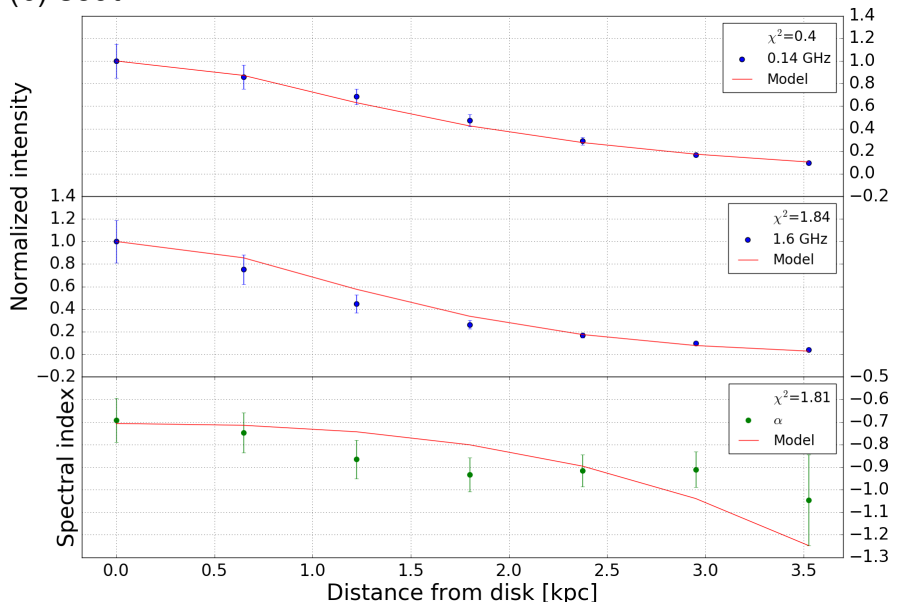

(d) north

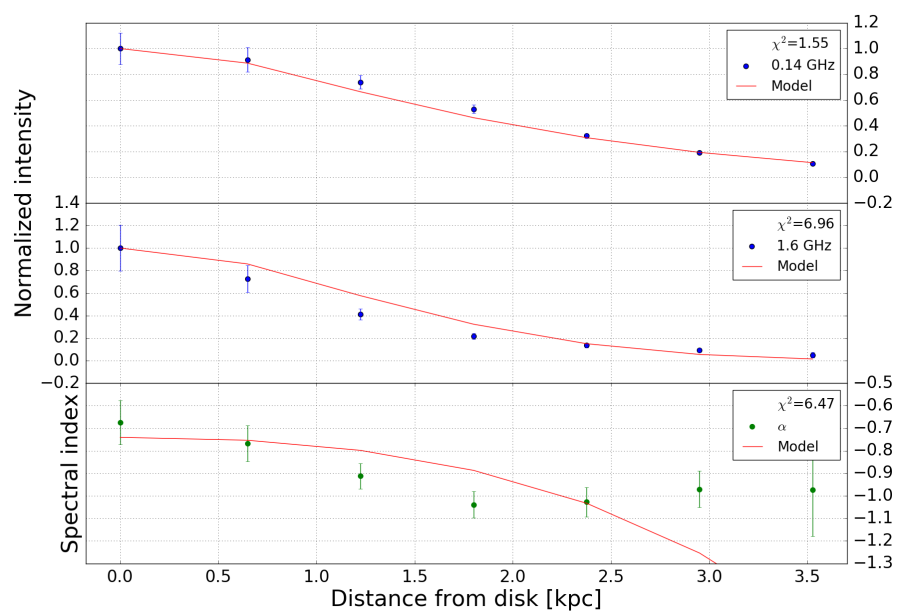

(d) south

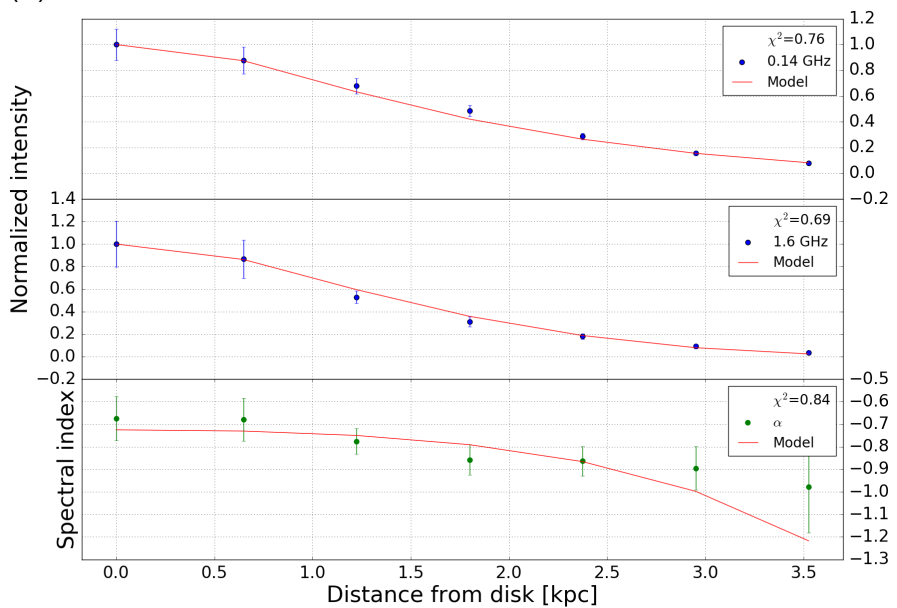

(b) south

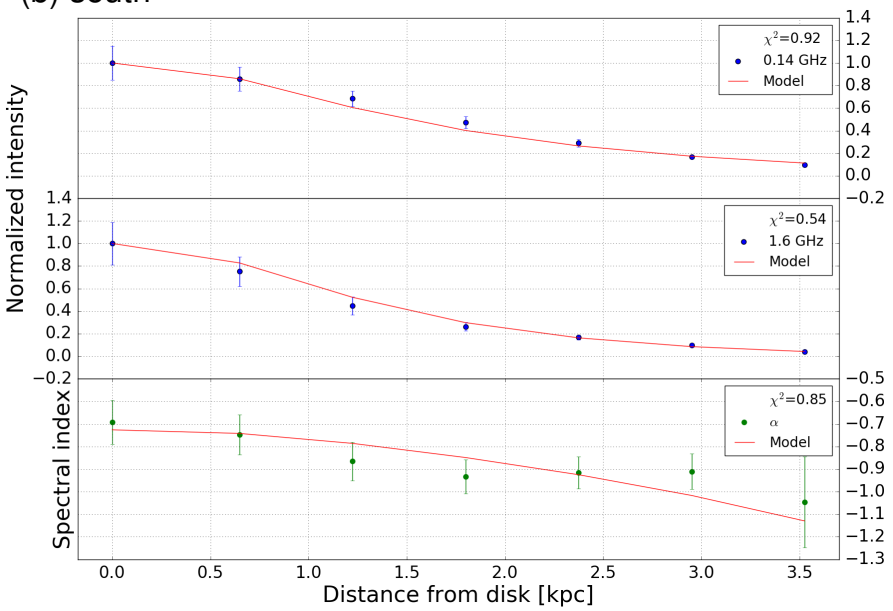

(d) north

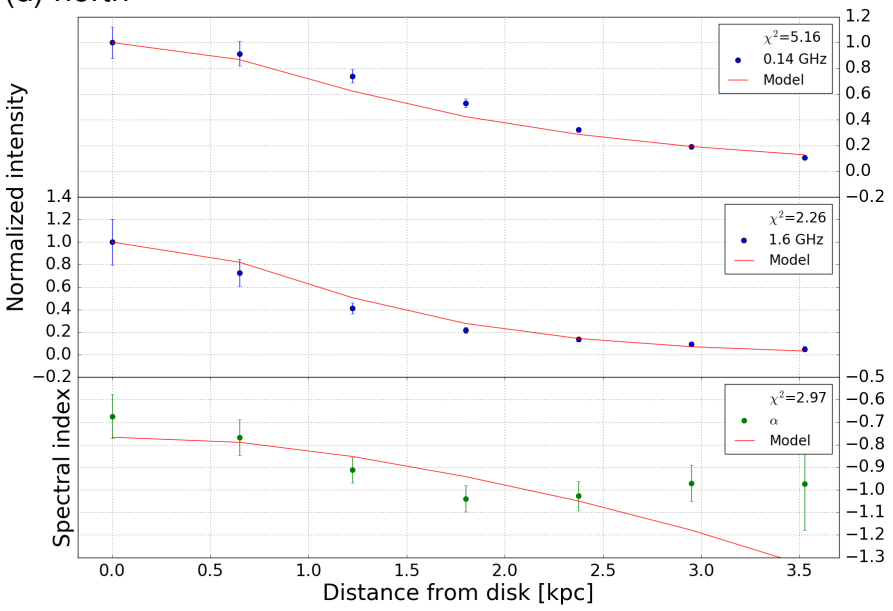

(d) south

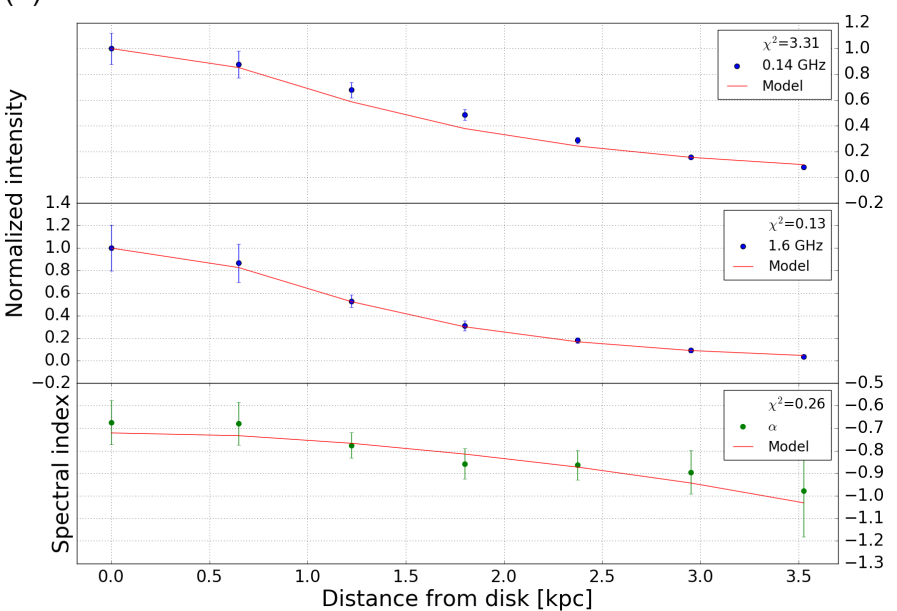

Fig. B.2. Best-fitting diffusion (left panels) and advection (right panels) models in strips b (south) and d (north and south). In each panel we show the vertical profiles. From top to bottom: $144 \mathrm{MHz}$ non-thermal intensities, the $1570 \mathrm{MHz}$ non-thermal intensities, and the non-thermal radio spectral index. Solid lines show the best-fitting models calculated with SPINNAKER. In each panel we also present the reduced $\chi_{\text {red }}^{2}$ for each observed profile and model profile. 


\section{Diffusion}

(e) north

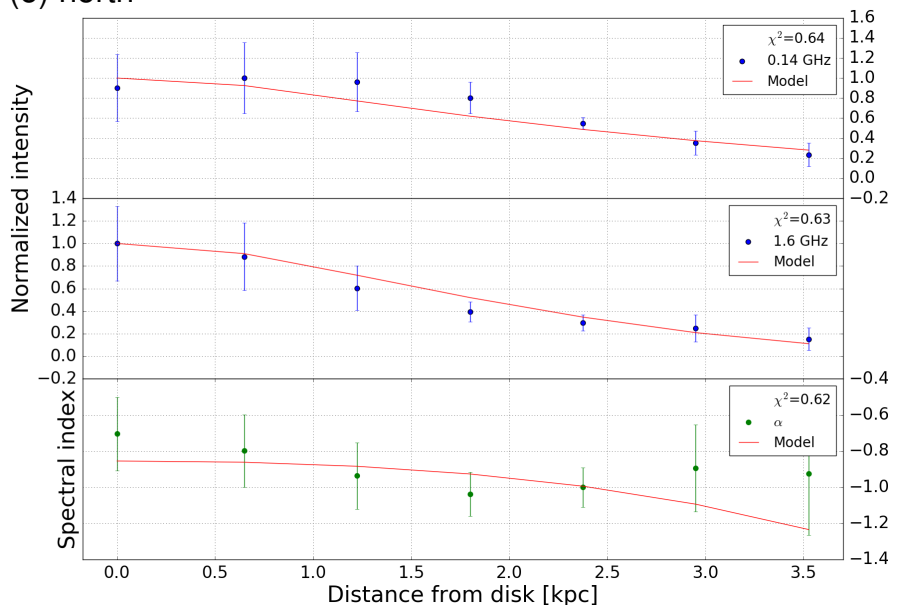

(e) south

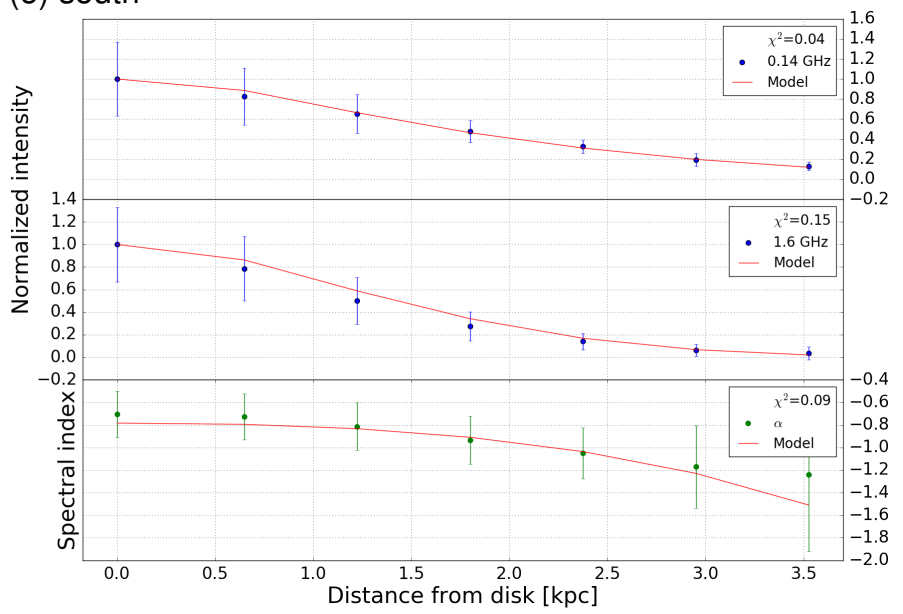

(e) north

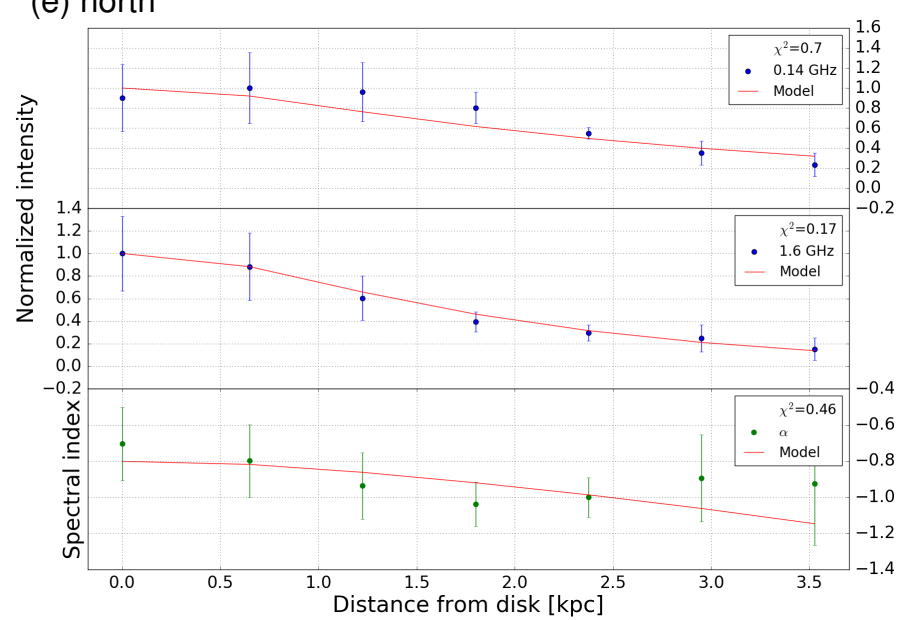

(e) south

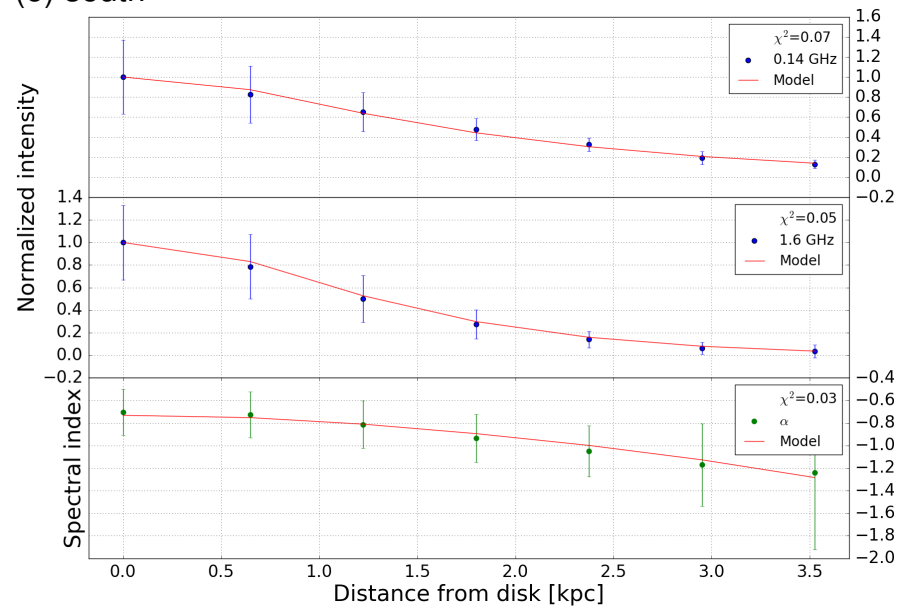

Fig. B.3. Best-fitting diffusion (left panels) and advection (right panels) models in strip e (north and south). In each panel we show the vertical profiles. From top to bottom: $144 \mathrm{MHz}$ non-thermal intensities, the $1570 \mathrm{MHz}$ non-thermal intensities, and the non-thermal radio spectral index. Solid lines show the best-fitting models calculated with SPINNAKER. In each panel we also present the reduced $\chi_{\text {red }}^{2}$ for each observed profile and model profile. 